\title{
Interobserver variability and aberrant E-cadherin immunostaining of lobular neoplasia and infiltrating lobular carcinoma
}

\author{
Young J Choi, Marguerite M Pinto, Liming Hao and Ali K Riba \\ Department of Pathology, Yale University School of Medicine, New Haven, CT, USA
}

\begin{abstract}
The distinction between lobular neoplasia and infiltrating lobular carcinoma from ductal neoplasia and infiltrating duct carcinoma with equivocal histologic features may present a challenge as this distinction has important therapeutic implications. Although E-cadherin staining has been of value in helping to make this determination, the variability of the E-cadherin staining pattern and the immunohistochemistry techniques can be problematic in clinical practice. A total of 161 cases of breast lesions previously diagnosed as lobular neoplasia and infiltrating lobular carcinoma were selected from the departmental files. Three surgical pathologists interpreted them in a blinded manner for the histology diagnoses and E-cadherin staining. E-cadherin staining was conducted on the paraffin-embedded sections of the breast lesions using two different source antibodies. Our results using morphology and E-cadherin stain agreed with the previous diagnoses of lobular neoplasia and infiltrating lobular carcinoma in 140 of 161 cases $(86.9 \%)$. Among the 140 cases, three pathologists agreed with the morphologic diagnoses of lobular neoplasia and infiltrating lobular carcinoma in $100(71.4 \%)$, two pathologists in $26(18.6 \%)$ and one pathologist in $14(10 \%)$. All three pathologists disagreed with the previous diagnoses of lobular neoplasia and infiltrating lobular carcinoma but reevaluated as ductal lesions in 21 cases (13.0\%). E-cadherin staining was confirmatory in 136 of total 161 cases $(84.5 \%)$ of both lobular and duct lesions by showing the loss of staining in lobular lesions and the presence of complete membrane staining in duct lesions. Aberrant E-cadherin reactions were retained weak or partial incomplete thin membrane reaction in lobular-type lesions and reduced membrane reaction in ductal-type lesions were seen in 25 of the total 161 cases (15.5\%). E-cadherin immunoreaction with two different antibodies showed discrepant results in 5 of 78 cases tested $(6.4 \%)$. This study illustrates (1) interobserver variability of the morphologic diagnoses of lobular neoplasia/infiltrating lobular carcinoma and duct neoplasia/infiltrating duct carcinoma, (2) the occasional presence of aberrant E-cadherin stain pattern in these breast lesions and (3) variability of E-cadherin immunostaining results by two different antibodies.
\end{abstract}

Modern Pathology (2008) 21, 1224-1237; doi:10.1038/modpathol.2008.106; published online 27 June 2008

Keywords: Iobular neoplasia; E-cadherin expression; aberrant immunoreaction; interobserver variability

Lobular neoplasia (atypical lobular hyperplasia and lobular carcinoma in situ) and infiltrating lobular carcinoma are distinct subsets of breast tumors, based on histology, genetics and biology. In 1941, Foote and Stewart ${ }^{1}$ described 'isolated cell or groups of small uniform cells in the lobule or in the terminal duct' to more extensive lesions with acinar distension and obliteration of the luminal space. Since then a number of authors ${ }^{2-6}$ have described generally accepted histologic criteria to distinguish lobular from ductal lesions of the breast. However, certain areas of morphologic overlap do

Correspondence: Dr YJ Choi, MD, Department of Pathology, Yale University School of Medicine, New Haven, CT 06520, USA.

E-mail: young.choi@yale.edu

Received 11 July 2007; revised 12 May 2008; accepted 18 May 2008; published online 27 June 2008 occur, such as (1) lobular carcinoma in situ may be mimicked by a solid growth pattern of low-grade duct carcinoma in situ and may coexist in the same duct lobular unit, $^{7}$ (2) comedo-type necrosis can occur in both duct carcinoma in situ and lobular carcinoma in situ, ${ }^{5,8}$ (3) pleomorphic lobular carcinoma in situ can be difficult to distinguish from duct carcinoma in $s i t u^{9,10}$ and (4) the diffuse targetoid growth pattern typified in classic lobular carcinoma may also be seen in both infiltrating duct carcinoma and infiltrating lobular carcinoma and its variants.

In recent years, E-cadherin immunostaining has been shown to be useful in distinguishing ductalfrom lobular-type breast lesions. E-cadherin is a cell adhesion molecule that interacts with the actin cytoskeleton by binding of $\alpha$-, $\beta$ - and $\gamma$-catenins. The loss of E-cadherin-mediated cell adhesion is thought 
to account for the characteristic dyscohesion of lobular neoplastic cells and highly infiltrative growth pattern of invasive lobular carcinomas. E-cadherin membrane staining is reported to be completely absent in lobular neoplasia/infiltrating lobular carcinoma and is present in duct carcinoma. However, the reduction of E-cadherin stain in some high-grade infiltrating duct carcinoma and retained weak E-cadherin staining in some lobular neoplasia/ infiltrating lobular carcinoma raise questions about using E-cadherin stain to distinguish lobular from duct lesions.

Although the treatment of stage-matched infiltrating lobular carcinoma and infiltrating duct carcinoma is similar and has similar outcomes, ${ }^{11-13}$ the distinction of in situ lesion is clinically important. Lobular carcinoma in situ diagnosed by core or excisional biopsy requires no further surgery, whereas duct carcinoma in situ necessitates complete surgical excision and possible additional radiotherapy.

The aims of this study are to (1) address the challenges in diagnosing lobular neoplasia/ infiltrating lobular carcinoma by morphology with routine hematoxylin and eosin stain and assess interobserver variability, (2) readdress the existence of aberrant E-cadherin staining in both duct and lobular lesions and (3) compare the E-cadherin immunoreaction using two different clone of antibodies.

\section{Materials and methods}

A total of 161 cases of lobular neoplasia/infiltrating lobular carcinoma lesions previously diagnosed during the period of 1998-2004 were retrieved from a pathology database. During this period, the total of 1497 cases of breast cancer were recorded in the hospital tumor registry and consisted of 807 (53\%) cases of infiltrating duct carcinoma NOS, 156 (10\%) duct carcinoma in situ, $98(6.5 \%)$ infiltrating lobular carcinoma, 44 (3.1\%) lobular carcinoma in situ and the rest other types.

Breast specimens were obtained from 40to 80-year-old female patients by core biopsy, excision or mastectomy. The tissue sections were prepared by fixation in $10 \%$ neutral-buffered formalin, processed and embedded in paraffin, and stained with hematoxylin and eosin. One pathologist selected one or two representative slides from each case, which contained the diagnostic lobular neoplasia/infiltrating lobular carcinoma lesions. Three other surgical pathologists each reviewed the hematoxylin and eosin slides independently in a blinded manner without the knowledge of the results of the E-cadherin immunostaining.

The morphology diagnoses of lobular neoplasia/ infiltrating lobular carcinoma were made by using published conventional architectural and cytologic criteria. $^{2-6}$ The terminal duct lobular units in classic lobular carcinoma in situ are distended by a solid proliferation of dyscohesive small cells with uniform round-oval nuclei showing relatively homogenous chromatin and absent or inconspicuous nuclei. These cells did not form tubules or lumen and consisted of the entire population of cells in the involved acinus. Some of the cells contained intracytoplasmic vacuoles, with occasional signet ring cells. Pleomorphic lobular carcinoma in situ is characterized by a growth pattern similar to the classic lobular carcinoma in situ but with a greater nuclear atypia. Infiltrating lobular carcinoma was classified as classic, solid, alveolar, tubulolobular and pleomorphic type. Tubulolobular carcinoma is composed of intermixed, round to angulated tubules and single-file cell cords with a diffuse and targeted pattern as in classic lobular carcinoma. The morphologic interpretation was grouped as (1) the ductal type including atypical duct hyperplasia, duct carcinoma in situ and infiltrating duct carcinoma, and (2) the lobular-type encompassing atypical lobular hyperplasia, lobular carcinoma in situ and infiltrating lobular carcinoma including its variants.

Immunohistochemistry was conducted on a $5-\mu \mathrm{m}$ thick formalin-fixed deparaffinized section by using E-cadherin antibodies from two different sources with their recommended appropriate linking system, but using the same heat-induced epitope antigen retrieval techniques: (1) 161 cases were stained with the E-cadherin antibody (ECH-6, prediluted; Cell Marque, Rocklin, CA, USA) using an LSAB2 linking system and (2) 78 cases also were stained with E-cadherin antibody (NCH-38, 1:25 dilution; DakoCytomation, Carpinteria, CA, USA) using an EnVision dual link system. All the stains were carried out by a Dako automated immunostainer. A positive control slide contained normal breast ducts and infiltrating duct breast carcinoma. An appropriate negative control was included. Only one section was tested from each case. In our laboratory, the immunoreactivity and the staining pattern with the E-cadherin antibody from Cell Marque (ECH-6 clone) was more reproducible and correlated with the morphologic features of the lobular neoplasia/infiltrating lobular carcinoma. Therefore, in this study, the E-cadherin immunoreaction with this antibody was used as the gold standard to interpret the reaction with the other antibody.

The presence of strong complete circumferential membrane stain in epithelial cells of normal ducts was defined as positive internal and external controls. The immunostaining intensity and pattern were evaluated as to the (1) absence or presence of circumferential membrane staining, or (2) reduced-intensity, partial or weak linear membrane staining, or focal or dot-like cytoplasmic or membrane reaction, or (3) a combination of these patterns. 
Table 1 E-cadherin immunoreaction in duct and lobular neoplasia (E-cadherin immunostaining)

\begin{tabular}{|c|c|c|c|c|c|c|c|}
\hline $\begin{array}{l}\text { Cases/ } \\
\text { diagnoses }\end{array}$ & $\begin{array}{l}\text { Complete } \\
\text { absence }\end{array}$ & $\begin{array}{l}\text { Weak/partial } \\
\text { fragmented }\end{array}$ & $\begin{array}{l}\text { Focal/dot-like } \\
\text { cytoplasm }\end{array}$ & $\begin{array}{l}\text { Complete } \\
\text { membrane }\end{array}$ & $\begin{array}{l}\text { Reduced/weak } \\
\text { membrane }\end{array}$ & $\begin{array}{l}\text { Antibody } \\
\text { clone/dilution }\end{array}$ & $\begin{array}{l}\text { IHC staining } \\
\text { technique }\end{array}$ \\
\hline 140 LN/ILC & $121(86.4 \%)$ & $16(11.5 \%)$ & $3(2.1 \%)$ & & & $\begin{array}{l}\text { ECH-6 clone } \\
\text { Cell Marque, } \\
\text { prediluted }\end{array}$ & $\begin{array}{l}\text { HIER } \\
\text { Citrate, pH } 6.0 \\
\text { EnVision Plus }\end{array}$ \\
\hline 21 DCIS/IDC & & & & $15(71.4 \%)$ & $6(28.6 \%)$ & & \\
\hline
\end{tabular}

CIS, carcinoma in situ; DCIS, duct carcinoma in situ; HIER, heat-induced epitope retrieval; IDC, infiltrating duct carcinoma; ILC, infiltrating lobular carcinoma.

Bold number and percentage: cases with aberrant E-cadherin reaction.

\section{Results}

Agreement with the previous diagnoses of lobular neoplasia/infiltrating lobular carcinoma lesions by morphology and E-cadherin staining was found in 140 of $161(86.9 \%)$ of the cases. Among the 140 cases, three pathologists agreed with the previous morphologic diagnoses of lobular neoplasia/ infiltrating lobular carcinoma in 100 cases $(71.4 \%)$, two pathologists in 26 cases (18.6\%) and one pathologist in 14 cases $(10.0 \%)$. All three pathologists disagreed with the previous diagnoses of lobular neoplasia/infiltrating lobular carcinoma but agreed with those as ductal-type lesion in 21 cases $(13.0 \%)$.

The 140 cases of lobular neoplasia/infiltrating lobular carcinoma consisted of 119 lobular neoplasia/infiltrating lobular carcinoma (106 cases of classic infiltrating lobular carcinoma and lobular carcinoma in situ and 13 lobular variants (9 cases of pleomorphic lobular carcinoma, 2 tubulolobular carcinoma and 1 each of solid carcinoma and alveolar-type lobular carcinoma)) and 21 cases that were initially interpreted as lobular lesion on morphology, but were reclassified as duct neoplasia/infiltrating duct carcinoma following E-cadherin staining.

As shown in Table 1, E-cadherin membrane expression supported the diagnoses of 121 of 140 cases $(86.4 \%)$ of lobular neoplasia/infiltrating lobular carcinoma by complete loss of membrane staining and 19 cases (13.6\%) with the classic morphology of lobular neoplasia/infiltrating lobular carcinoma but the aberrant staining by retained or weak thin membrane staining (Figures 1 and 2). The 121 lobular neoplasia/infiltrating lobular carcinomas included 106 cases that were initially diagnosed as lobular neoplasia/infiltrating lobular carcinoma and an additional 15 cases that were diagnosed as lobular neoplasia/infiltrating lobular carcinoma after E-cadherin staining. Of nine pleomorphic variants, eight showed complete absence and one focal weak staining. Both solid and alveolar types showed complete absence of membrane staining. Of two cases of tubulolobular carcinoma, one showed complete absence and the other reduced incomplete linear membrane staining. The seven- teen cases of combined lobular and duct lesions included five lobular carcinoma in situ with duct carcinoma in situ in the same lobule, nine lobular carcinoma in situ with infiltrating duct carcinoma, one infiltrating lobular carcinoma with duct carcinoma in situ and two lobular carcinoma in situ with duct carcinoma in situ and infiltrating duct carcinoma. E-cadherin stain in these cases showed transition from weak to strong and focal to complete membrane staining in the in situ and also invasive carcinoma (Figure 3).

The 19 aberrant E-cadherin staining (Table 1; Figure 4) of lobular neoplasia/infiltrating lobular carcinoma was exhibited as fragmented, discontinuous, faint, partial membrane or granular staining in 16 cases $(11.5 \%)$ and perinuclear dot-like or punctate cytoplasmic staining in 3 cases $(2.1 \%)$, and that of 6 duct lesions $(28.6 \%)$ as reduced weak linear but thin complete membrane staining. The greatest numbers of strongly E-cadherin-stained lobular carcinoma cells were identified near the periphery, particularly along the leading edge of the invasive tumors. In lobules partially occupied by lobular carcinoma in situ cells, the tumor cell population was outlined by its negativity for E-cadherin staining, contrasting with the positive staining of the residual terminal ductal epithelium.

Of the 21 cases that were reevaluated as duct lesion by the morphology, the E-cadherin staining confirmed the diagnoses of duct lesions by complete circumferential membrane expression throughout the tumor in 15 of 21 cases (71.4\%; Table 1; Figure 2), and aberrant staining with reduced or focal membrane reaction in 6 cases $(28.6 \%$; Figure 4$)$. The reduced or focal aberrant staining was mostly located at the peripheral region or the leading edge of invasion of infiltrating duct carcinoma or invasive area adjacent to the duct carcinoma in situ. From strong to weak heterogenous staining was observed even within the same duct or within the same lobules (Figure 4). These neoplastic cells with the reduced or focal reactive ducts appear to have greater cellular dyscohesion and a more extensive single-cell infiltrative pattern than cells located in the central regions of the neoplasm.

In our study, we observed the following pertinent findings: E-cadherin staining was confirmatory in 
136 of total 161 cases (84.5\%) of both lobular lesions by showing the loss of membrane staining in lobular lesions and by the presence of complete membrane staining in duct lesions. The aberrant E-cadherin reaction was seen in 25 of 161 cases (15.5\%) by combining 19 cases of lobular neoplasia/infiltrating lobular carcinoma and 6 cases of duct lesions. When the aberrant E-cadherin expression was tested by another E-cadherin antibody (Dako, NCH-38 clone) and its linking system, E-cadherin immunoreaction was discrepant in 5 of 78 cases $(6.4 \%)$. This indicates that the variability and the frequency of the aberrant E-cadherin expression in published reports from different laboratories may be due to the variability of the immunohistochemistry testing system.

As a whole, our study showed the usefulness of E-cadherin staining in distinguishing lobular neoplasia/infiltrating lobular carcinoma from duct lesions with the equivocal morphologic features.
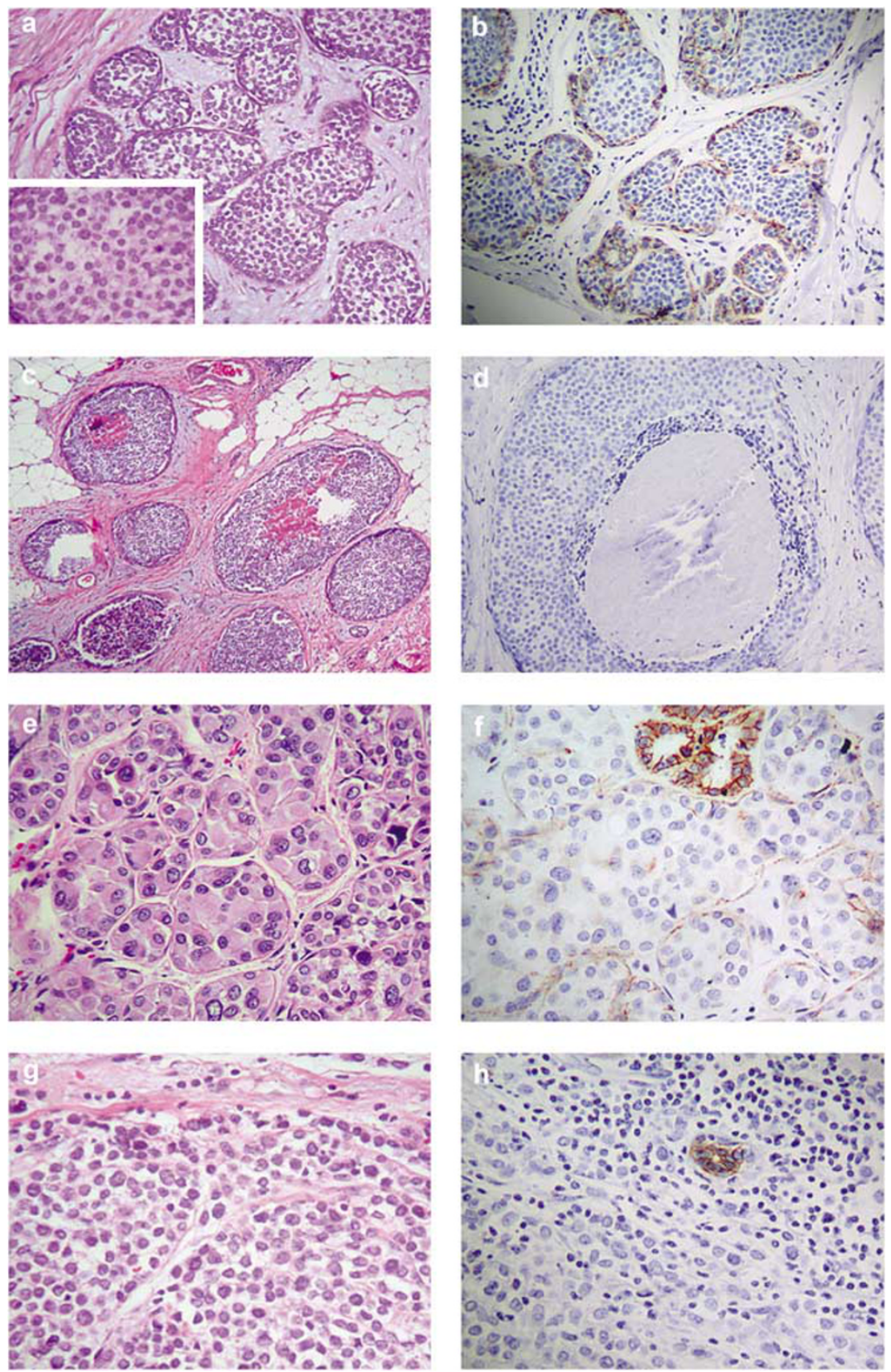

Figure 1 Diagnostic utilization of E-cadherin staining in lobular-type lesions. Complete loss of E-cadherin membrane staining in classic lobular carcinoma in situ with small and round cells with mild hyperchromasia and inconspicuous nucleoli, occasional intracytoplasmic lumina and rare signet-ring morphology that distend the lobular unit (a, b), lobular carcinoma in situ with central necrosis (c, d), pleomorphic lobular carcinoma in situ (e, f), solid lobular carcinoma (g, h), classic infiltrating lobular carcinoma (i, j), infiltrating pleomorphic carcinoma $(\mathbf{k}, \mathbf{l})$. 

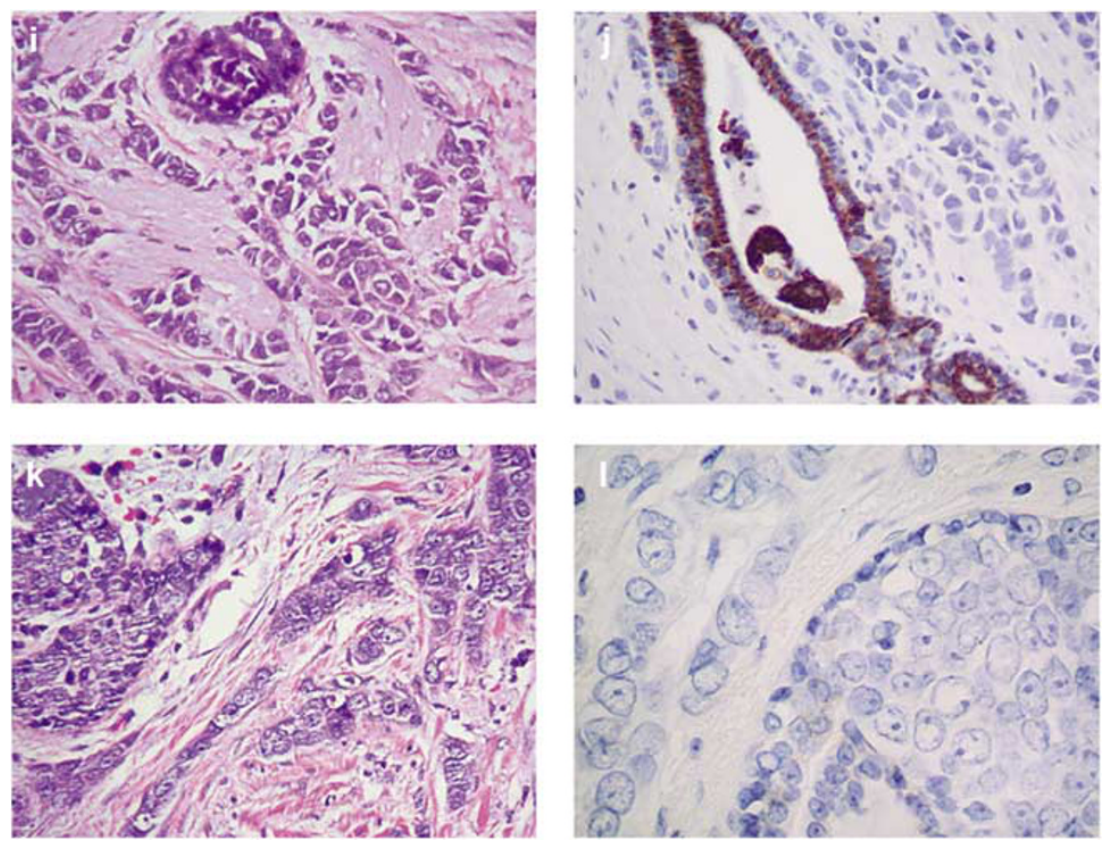

Figure 1 Continued.

These were solid duct carcinoma in situ vs lobular carcinoma in situ (11), sclerosing adenosis involved by lobular carcinoma in situ vs duct carcinoma in situ (1), pagetoid spread of duct carcinoma vs lobular carcinoma (1), pleomorphic lobular carcinoma in situ and infiltrating lobular carcinoma vs infiltrating duct carcinoma (3) or combined tumors as illustrated above. In the majority of cases, E-cadherin-negative in situ lesions composed of a low-grade uniform solid proliferation, regardless of the predominant site of involvement (ducts or lobules), were usually accompanied by an invasive carcinoma with the features of E-cadherin-negative infiltrating lobular carcinoma. Conversely, E-cadherinpositive in situ lesions were accompanied by E-cadherin-positive invasive carcinoma of the ductal type. Neither the presence of necrosis nor that of an occasional microacini would exclude a lobular carcinoma in situ.

\section{Discussion}

Our study illustrates the challenges for rendering an accurate interpretation of lobular neoplasia/infiltrating lobular carcinoma by morphology even with the aid of E-cadherin staining. Some of the difficulties in distinguishing lobular neoplasia/infiltrating lobular carcinoma from duct lesions arise from overlapping morphologic features, resulting in inter- and intraobserver variability and subjectivity.

In recent years, E-cadherin staining has been utilized routinely to distinguish indeterminate morphologic features of duct vs lobular lesions (Table 2). However, our study demonstrates the variability of E-cadherin staining and an aberrant
E-cadherin staining pattern in both duct and lobular lesions, making it difficult to interpret accurately lobular neoplasia/infiltrating lobular carcinoma. A strong uniform cell membrane staining was displayed in some tumor cells, suggesting a duct origin of the tumor cells, but they also displayed a lobular growth pattern, a 'lobular' phenotype. Other tumor cells exhibited a reduced discontinuous thin or punctate membrane staining but a ductal-type morphology. Thus, weak incomplete positive membrane staining may not completely exclude the diagnoses of infiltrating lobular carcinoma or include of infiltrating duct carcinoma as E-cadherin expression is retained in some cases with a characteristic infiltrating lobular carcinoma morphology and reduced in some cases with infiltrating duct carcinoma. In addition, weak dot-like cytoplasm or discontinuous membrane reaction can be seen in the morphology of both lobular- or ductaltype lesions. Similarly, the morphology of duct carcinoma in situ with comedo-type necrosis can be either duct carcinoma in situ or lobular carcinoma in situ, although the absence of E-cadherin staining and the appropriate morphologic changes of lobular neoplasia provides a reasonable degree of confidence that the lesion is lobular neoplasia. ${ }^{8,21}$

The pagetoid spread of lobular carcinoma in situ to E-cadherin-reactive benign cells could be mistaken for the reactivity of duct carcinoma in situ. These phenomena may account for the reported instances of lobular carcinoma with E-cadherin reactivity. The complete absence of staining adjacent to the lesion with faint partial staining in the same lobule with indeterminate histology type may be in fact combined duct and lobular lesions that require different patient management. 

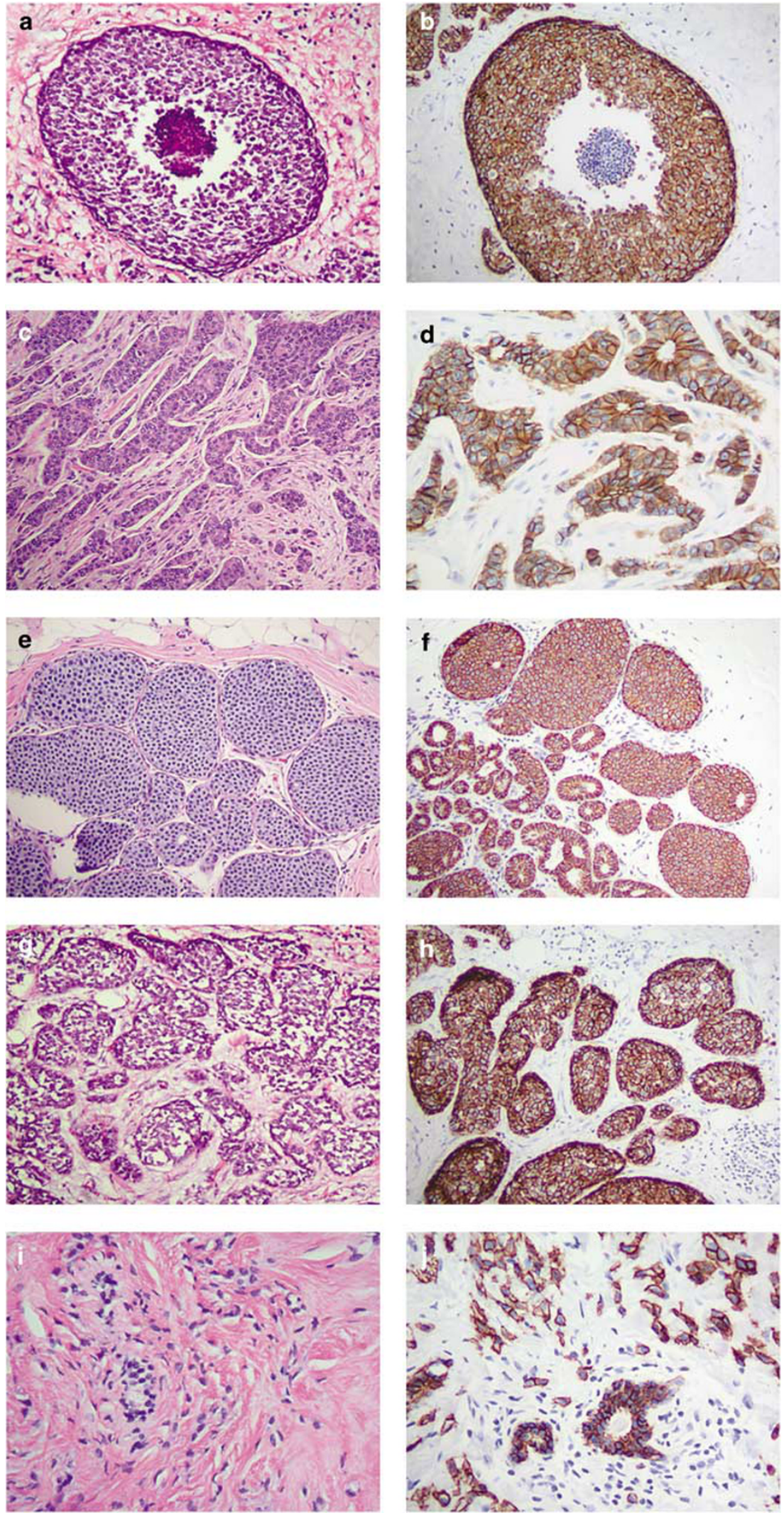

Figure 2 Diagnostic utilization of E-cadherin staining in duct-type lesions. Complete circumferential membrane of E-cadherin staining: classic duct carcinoma in situ (a, b), classic infiltrating duct carcinoma (c, d), carcinoma in situ with lobular configuration but complete membrane staining $(\mathbf{e}, \mathbf{f})$, in situ duct carcinoma with lobular configuration and dyscohesive low-grade tumor cells but complete membrane staining $(\mathbf{g}, \mathbf{h})$ and infiltrating carcinoma with targetoid spread but complete membrane staining (i, j). 

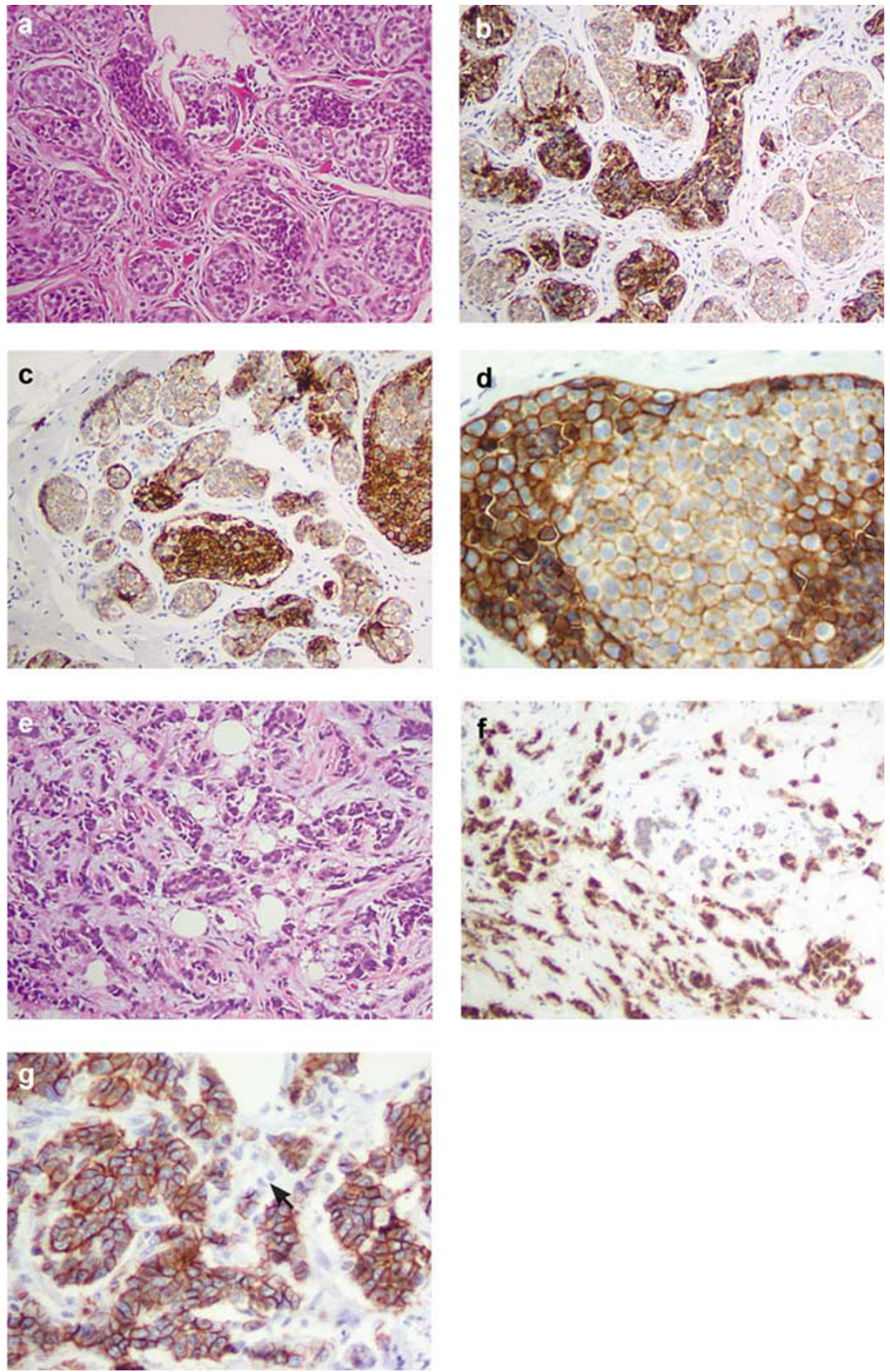

Figure 3 Combined lobular and duct carcinoma. The morphologic features of lobular and duct growth patterns and mixed E-cadherin staining patterns of absent, weak to strong complete membrane staining with transition from strong membrane to granular staining in the same lobules or in the same ducts. The E-cadherin staining patterns were similar in both in situ (a-d) and invasive lesions (e-g). Arrow indicates a loss of E-cadherin staining infiltrating cancer cells.

As shown in Table 3, such aberrant E-cadherin reaction has been observed by previous studies in the ranges of $0.4-54 \%$. Goldstein et $a 2^{24}$ reported $9 \%$ of lobular carcinoma in situ with E-cadherin membrane expression, usually in a patchy distribution and of lesser intensity than noted in duct carcinoma in situ, and that the patients with E-cadherin reactive lobular carcinoma in situ developed a subsequent ipsilateral carcinoma significantly more often than patients with E-cadherin nonreactive lobular carcinoma in situ. They suggested reactive E-cadherin in lobular carcinoma in situ as risk factors for subsequent carcinoma similar to those of patients with low-grade intraductal carcinoma. In addition, others reported reduced E-cadherin reaction as a predictor of increased invasiveness, disease recurrence and metastatic potential in high-grade duct carcinoma, ${ }^{18,29,32-35}$ and retained partial thin granular heterogenous membrane or cytoplasmic E-cadherin expression in lobular-type lesions. ${ }^{11,14,19,22,24,27,29-31,36}$ Focal cytoplasmic reaction was considered to be related to the presence of $\mathrm{P}$ - or $\mathrm{N}$-cadherin. ${ }^{37}$ 
Although loss of E-cadherin-mediated cell adhesion is thought to account for the characteristic dyscohesion of lobular neoplasia and the infiltrative growth pattern of invasive lobular carcinoma, lobular neoplasia/infiltrating lobular carcinoma also has been shown to have alterations of the E-cadherin complex, $\alpha$-catenin, $\beta$-catenin and p120 catenin. ${ }^{20,38-43}$ Thus, the aberrant staining in some cases may be due to alterations not only in E-cadherin protein but also in the catenins.
The genetic mutations and loss of heterozygosity ${ }^{40,44-48}$ involving a region of the $16 q$ chromosome, defects in transcription ${ }^{49}$ and methylation $^{47,50,51}$ have been demonstrated in lobular neoplasia/infiltrating lobular carcinoma. Such changes of the E-cadherin gene are thought to be responsible for the loss of E-cadherin membrane expression and it appears to be an early event in the majority of lobular carcinoma in situ cases. ${ }^{52}$ Compiled data from multiple studies ${ }^{46}$ revealed that
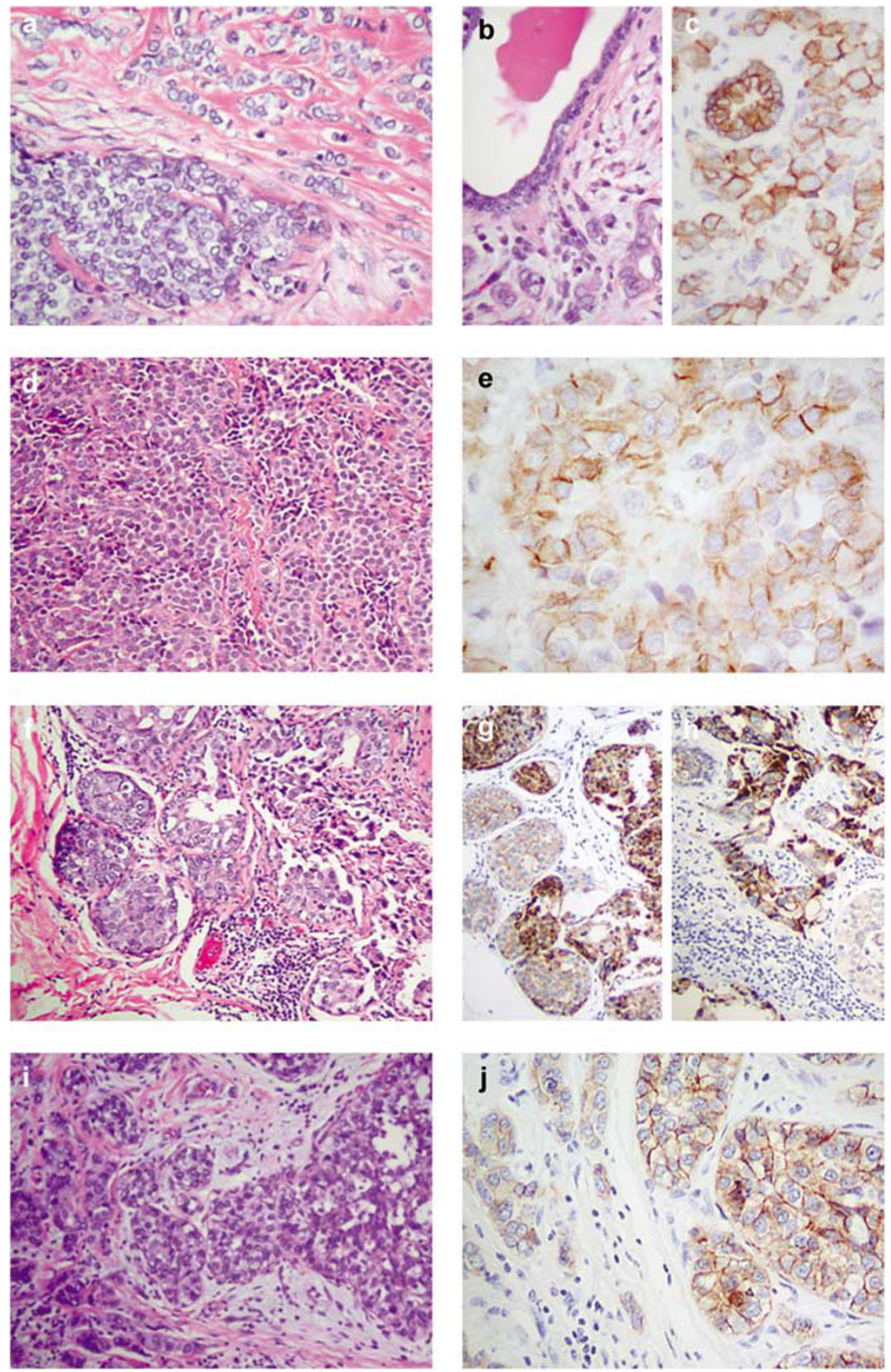

Figure 4 Aberrant E-cadherin staining with retained staining in lobular carcinoma and reduced and weak staining in duct-type lesions. Infiltrating lobular carcinoma with retained beaded, discontinuous, faint, partial granular or sparse membrane staining and perinuclear dot-like cytoplasmic staining (a-c), lobular carcinoma, alveolar variant with retained staining (d, e); reduced partial and focal membrane staining in DCIS and infiltrating duct carcinoma $(\mathbf{f}, \mathbf{g} / \mathbf{h}, \mathbf{i}, \mathbf{j})$, tubulolobular carcinoma with reduced weak staining (k-n) and infiltrating carcinoma with neuroendocrine features and reduced and focally accentuated staining $(\mathbf{o}, \mathbf{p})$. 

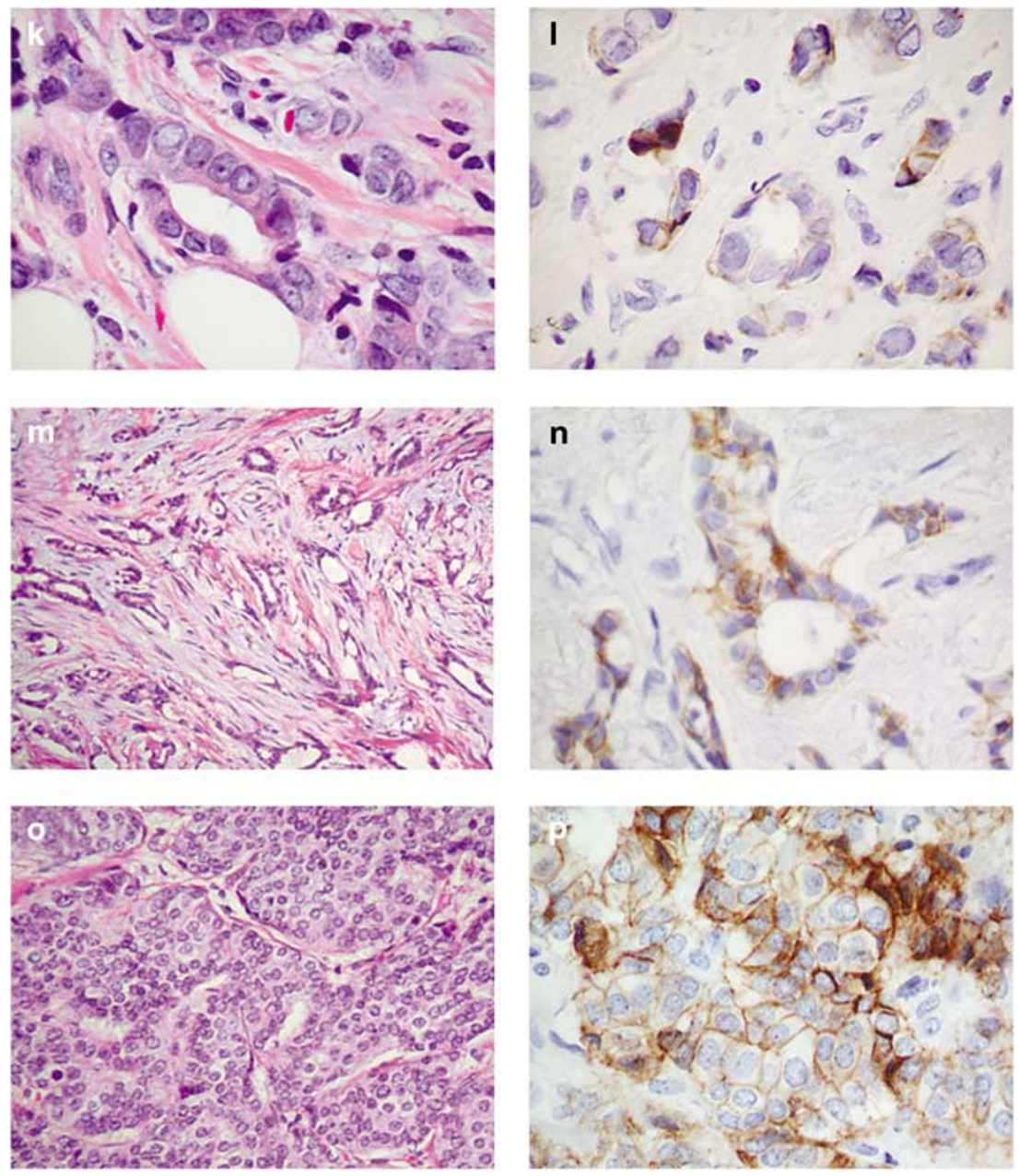

Figure 4 Continued.

77 of 247 (31\%) invasive lobular carcinomas and 27 of $40(68 \%)$ lobular carcinomas in situ harbored E-cadherin gene mutations and 40 of 72 (56\%) invasive lobular carcinomas have shown evidence of promotor methylation. Loss of heterozygosity for the chromosomal region containing the E-cadherin gene was detected in 138 of 174 (79\%) infiltrating lobular carcinoma cases and 17 of 29 (59\%) lobular carcinoma in situ cases. Asgeirsson et al ${ }^{23}$ reported loss of heterozygosity in $46 \%$ of infiltrating duct carcinoma cases in comparison with $89 \%$ in infiltrating lobular carcinoma, and loss of heterozygosity in infiltrating duct carcinoma was associated with heterogenous E-cadherin staining in $71 \%$.

The above information suggests that the structural phenotype does not always match the 'genotype' as illustrated by the E-cadherin immunohistochemical staining pattern. The retained aberrant E-cadherin expression in lobular lesions may imply the presence of an intact $16 \mathrm{q}$ E-cadherin gene with a duct carcinoma in situ genotype, with its accompanying risk factors of duct carcinoma in situ. Also, the characteristic cellular dyscohesion of lobular carcinoma in situ may be the result of low-grade duct carcinoma in situ that has sustained alterations in one of the molecules related to the E-cadherin binding system, such as the $\alpha-, \beta$ or $\gamma$-catenins but an intact E-cadherin molecule. Conversely, the reduced E-cadherin expression in duct lesions may be due to downregulation and decreased E-cadherin gene transcription caused by hypermethylation, ${ }^{46}$ or post-translational modification and abnormal function of the normally expressed molecules. Downregulation of $\gamma$-catenin can affect the formation of desmosomes, suggesting that cell dyscohesion in some ductal carcinoma may be due to dysfunction of cell-binding structures other than adherent junctions. In addition, a gene other than the E-cadherin may be the target in duct carcinoma. ${ }^{48}$

As shown in Table 3, the degree and frequency of aberrant E-cadherin staining is variable in different studies. This may well be a reflection of variability of immunohistochemistry techniques including the number of sections stained per case, thickness of the sections, clones of the antibody used and antigen retrieval system. Most of the studies reported used the HECD-1 clone of E-cadherin but different dilutions, antigen retrieval system, buffer, $\mathrm{pH}$, incubation time and linking system. It is interesting 
Table 2 Diagnostic utilization of E-cadherin immunostaining in distinguishing duct vs lobular neoplasia (E-cadherin immunoreaction)

\begin{tabular}{|c|c|c|c|c|c|c|c|}
\hline Publication & Cases/diagnoses & $\begin{array}{l}\text { Complete } \\
\text { absence }\end{array}$ & $\begin{array}{l}\text { Complete } \\
\text { membrane }\end{array}$ & $\begin{array}{l}\text { Reduced/ } \\
\text { partial }\end{array}$ & Focal & $\begin{array}{l}\text { Antibody } \\
\text { clone/dilution }\end{array}$ & IHC staining technique \\
\hline Siitonen et $a l^{14}$ & 55 ILC & $55(100 \%)$ & - & - & - & $\begin{array}{l}\text { HECD-1 } \\
1: 800\end{array}$ & $\begin{array}{l}\text { Overnight incubation } \\
\text { at } 4{ }^{\circ} \mathrm{C} \\
\text { PBS buffer } \\
\text { ABC }\end{array}$ \\
\hline \multirow[t]{2}{*}{ Acs et $a l^{15}$} & 42 ILC & $41(98 \%)$ & - & - & $1(2 \%)$ & $\begin{array}{l}\text { HECD-1 } \\
1: 200\end{array}$ & $\begin{array}{l}\text { Overnight incubation } \\
\text { at } 4^{\circ} \mathrm{C} \\
\text { Citrate, pH } 6 \\
\text { Strep-HRP }\end{array}$ \\
\hline & $\begin{array}{l}53 \text { LCIS } \\
100 \text { IDC } \\
131 \text { DCIS }\end{array}$ & $50(94 \%)$ & $\begin{array}{c}- \\
96(96 \%) \\
128(97.7 \%)\end{array}$ & $\begin{array}{c}- \\
4(4 \%) \\
3(2.3 \%)\end{array}$ & $3(6 \%)$ & & \\
\hline \multirow[t]{2}{*}{ Goldstein et $a l^{16}$} & 37 DCIS & & $37(100 \%)$ & - & & $\begin{array}{l}4 \mathrm{~A} 2 \mathrm{C}^{\mathrm{b}} \\
1: 50\end{array}$ & $\begin{array}{l}\text { HIER (steamer) } \\
\text { EDTA, pH } 7 \\
\text { EnVision Plus }\end{array}$ \\
\hline & $\begin{array}{l}22 \text { LCIS with necrosis } \\
19 \text { indeterminate CA }\end{array}$ & $\begin{array}{l}22(100 \%) \\
13(68.4 \%)\end{array}$ & $6 \stackrel{-}{(31.6 \%)}$ & $\begin{array}{l}- \\
-\end{array}$ & $\begin{array}{l}- \\
-\end{array}$ & & \\
\hline Wheeler et $a^{17}$ & 27 tubulolobular CA & & $27(100 \%)$ & - & - & $\begin{array}{l}\text { 4A2 C7 } \\
1: 40\end{array}$ & $\begin{array}{l}\text { HIER (pressure } \\
\text { cooker) } \\
\text { ABC }\end{array}$ \\
\hline Rakha et $a 1^{18}$ & 1516 nonlobular CA & $109(7.2 \%)$ & $1407(92.8 \%)$ & - & - & $\begin{array}{l}\text { HECD-1 } \\
1: 200\end{array}$ & $\begin{array}{l}\text { HIER (microwave) } \\
\text { Citrate, pH } 6 \\
\text { ABC }\end{array}$ \\
\hline \multirow[t]{2}{*}{ Qureshi et $a l^{19}$} & 4 tubulolobular CA & - & $4(100 \%)$ & - & - & $\begin{array}{l}4 \mathrm{~A} 2 \mathrm{C} 7 \\
1: 400\end{array}$ & $\begin{array}{l}\text { HIER (hot plate) } \\
\text { EDTA, pH } 8 \\
\text { ABC }\end{array}$ \\
\hline & $\begin{array}{l}9 \text { invasive CA, NOS } \\
49 \text { ILC } \\
204 \text { IDC }\end{array}$ & $\begin{array}{c}4(44.4 \%) \\
44(90 \%) \\
1(0.5 \%)\end{array}$ & $\begin{aligned} 5 & (55.6 \%) \\
5 & (10 \%) \\
203 & (99.5 \%)\end{aligned}$ & $\begin{array}{l}- \\
- \\
-\end{array}$ & $\begin{array}{l}- \\
- \\
-\end{array}$ & & \\
\hline \multirow[t]{2}{*}{ Kuroda et $a l^{20}$} & 16 tubulolobular CA & $4(25 \%)$ & $12(75 \%)$ & - & - & $\begin{array}{l}\text { NCH- }{ }^{\mathrm{c}} \\
1: 50\end{array}$ & $\begin{array}{l}\text { HIER (pressure cooker) } \\
\text { Citrate, pH } 6 \\
\text { (unknown linking } \\
\text { system) }\end{array}$ \\
\hline & 20 ILC & $20(100 \%)$ & - & - & - & & \\
\hline
\end{tabular}

ABC, avidin-biotin complex; CA, carcinoma; CIS, carcinoma in situ; DCIS, duct carcinoma in situ; HIER, heat-induced epitope retrieval; HRP, horseradish peroxidase; IDC, infiltrating duct carcinoma; ILC, infiltrating lobular carcinoma.

Sources of E-cadherin antibodies: a and b, Zymed, San Francisco, CA, USA; c, Dako, Carpinteria, CA, USA. 
Table 3 Aberrant E-cadherin immunoreaction in duct and lobular neoplasia (E-cadherin immunostaining)

\begin{tabular}{|c|c|c|c|c|c|c|c|}
\hline Publication & Cases/diagnoses & $\begin{array}{l}\text { Complete } \\
\text { absence }\end{array}$ & $\begin{array}{l}\text { Complete } \\
\text { membrane }\end{array}$ & Reduced/partial & Focal & $\begin{array}{l}\text { Antibody } \\
\text { clone/dilution }\end{array}$ & $\begin{array}{l}\text { IHC staining } \\
\text { technique }\end{array}$ \\
\hline Moll et $a 2^{22}$ & 41 IDC & - & $19(46 \%)$ & $22(54 \%)$ & - & $6 \mathrm{~F}^{\mathrm{a}}$ & $\begin{array}{l}\text { HIER (microwave) } \\
\text { Citrate buffer } \\
\text { ABC }\end{array}$ \\
\hline Siitonen et $a l^{14}$ & $\begin{array}{l}22 \text { ILC } \\
239 \text { IDC }\end{array}$ & $19(86.4 \%)$ & $115(-\overline{48} \%)$ & $124_{(52 \%)}^{-}$ & $\begin{array}{c}3(13.6 \%) \\
-\end{array}$ & $\begin{array}{l}\text { HECD-1 } \\
1: 800\end{array}$ & $\begin{array}{l}\text { Overnight at } 4^{\circ} \mathrm{C} \\
\text { PBS buffer } \\
\text { ABC }\end{array}$ \\
\hline Asgeirsson et $a l^{23}$ & $\begin{array}{l}20 \text { DCIS } \\
97 \text { IDC }\end{array}$ & $18 \stackrel{-}{(19 \%)}$ & $\begin{array}{l}16(80 \%) \\
24(25 \%)\end{array}$ & $\begin{array}{r}4(20 \%) \\
55(56 \%)\end{array}$ & $\begin{array}{l}- \\
-\end{array}$ & $\begin{array}{l}5 \mathrm{H} 9^{\mathrm{c}} \\
1: 10\end{array}$ & $\begin{array}{l}\text { HIER (microwave) } \\
\text { Citrate, pH } 6 \\
\text { HRP }\end{array}$ \\
\hline Acs et $a l^{15}$ & $\begin{array}{l}14 \text { ILC } \\
100 \text { IDC }\end{array}$ & $\begin{array}{c}9(64 \%) \\
-\end{array}$ & $\begin{array}{c}1(\mathbf{7} \%) \\
96(96 \%)\end{array}$ & $4 \overline{(4 \%)}$ & $\begin{array}{c}4(29 \%) \\
-\end{array}$ & $\begin{array}{l}\text { HECD-1 } \\
1: 200\end{array}$ & $\begin{array}{l}\text { Overnight at } 4^{\circ} \mathrm{C} \\
\text { Citrate, pH } 6 \\
\text { Strep-HRP }\end{array}$ \\
\hline & $\begin{array}{l}42 \text { ILC } \\
53 \text { LCIS } \\
131 \text { DCIS }\end{array}$ & $\begin{array}{l}41(98 \%) \\
50(94 \%)\end{array}$ & $\begin{array}{c}1(\mathbf{2} \%) \\
- \\
128(97.7 \%)\end{array}$ & $\begin{array}{c}- \\
- \\
3(2.3 \%)\end{array}$ & $3(6 \%)$ & & \\
\hline Goldstein et a $1^{16}$ & 22 LCIS & $20(91 \%)$ & - & - & $2(9 \%)$ & $\begin{array}{l}4 \mathrm{~A} 2 \mathrm{C}^{\mathrm{d}} \\
1: 500\end{array}$ & $\begin{array}{l}\text { HIER (microwave) } \\
\text { Citrate, pH } 6 \\
\text { HRP }\end{array}$ \\
\hline Goldstein et $a 2^{24}$ & 82 ILC & $73(89.1 \%)$ & - & - & $9(10.9 \%)$ & $\begin{array}{l}\text { 4A2 C7 } \\
1: 50\end{array}$ & $\begin{array}{l}\text { HIER (steamer) } \\
\text { EDTA, pH } 7 \\
\text { EnVision Plus }\end{array}$ \\
\hline Maluf et $a 1^{25}$ & 12 indeterminate CIS & $5(41.6 \%)$ & $5(41.6 \%)$ & - & $2(16.8 \%)$ & $\begin{array}{l}\text { HECD-1 } \\
1: 100\end{array}$ & $\begin{array}{l}\text { HIER } \\
\text { Citrate, pH } 6 \\
\text { LSAB+ link }\end{array}$ \\
\hline Jacobs et $a l^{26}$ & 17 indeterminate CIS & $6(35.3 \%)$ & $5(29.4 \%)$ & - & $6(35.3 \%)$ & $\begin{array}{l}\text { HECD-1 } \\
1: 500\end{array}$ & $\begin{array}{l}\text { HIER (steamer) } \\
\text { Citrate, pH } 6 \\
\text { ABC }\end{array}$ \\
\hline & $\begin{array}{l}33 \text { DCIS } \\
28 \text { LCIS }\end{array}$ & $28(\overline{-})$ & $33(100 \%)$ & $\begin{array}{l}- \\
-\end{array}$ & $\begin{array}{l}- \\
-\end{array}$ & & \\
\hline Goldstein $^{27}$ & 143 ILC & $136(95 \%)$ & 7 (5\%) & - & - & $\begin{array}{l}\text { HECD-1 } \\
1: 100\end{array}$ & $\begin{array}{l}\text { HIER (steamer) } \\
\text { EDTA, pH } 7.5 \\
\text { EnVision Plus }\end{array}$ \\
\hline Wahed et $a l^{28}$ & 14 pleomorphic ILC & $12(86 \%)$ & - & - & $2(14 \%)$ & 4A2 C7 & $\begin{array}{l}\text { No Ag retrieval } \\
\text { ABC }\end{array}$ \\
\hline Kowalski et $a 2^{29}$ & 22 IDC & $12(55 \%)$ & - & $10(45 \%)$ & - & $\begin{array}{l}\text { HECD-1 } \\
\text { (dil, unknown) }\end{array}$ & $\begin{array}{l}\text { HIER (pressure cooker) } \\
\text { Citrate, pH } 6 \\
\text { (unknown linking system) }\end{array}$ \\
\hline & 8 ILC & - & - & - & $\begin{array}{l}\mathbf{8}(\mathbf{1 0 0} \%) \\
\text { cytoplasm staining }\end{array}$ & & \\
\hline Kovacs et $a l^{30}$ & 82 IDC & $4(4.8 \%)$ & $78(95.2 \%)$ & - & & $\begin{array}{l}\text { HECD-1 } \\
1: 150\end{array}$ & $\begin{array}{l}\text { HIER (pressure cooker) } \\
\text { Citrate, pH } 6.5 \\
\text { ABC }\end{array}$ \\
\hline
\end{tabular}

16 ILC

cytoplasm staining 
to note that Goldstein et $a l^{24}$ found that the E-cadherin reactivity varied according to the number of blocks and thickness of the section. In relation to the different clone and source of the antibodies, our study showed a 6.4\% discrepancy between two different antibodies. Different clones of E-cadherin antibody may fail to react in a membranous pattern and occasionally may demonstrate diffuse cytoplasmic or dot-like weak immunoreactivity with human P-cadherin. ${ }^{30}$ The observations suggest that the immunohistochemistry for E-cadherin staining requires standardization.

Although infiltrating lobular carcinoma and infiltrating duct carcinoma are different entities with different clinical courses and different biologic phenotypes, no clinically meaningful differences in survival are evident. At present both types are managed similarly and the histologic subtypes are not a factor in the therapeutic decision-making process and are not considered an important prognostic or predictive factor at diagnoses. However, Cristofanilli et $a l^{53}$ reported that classic infiltrating lobular carcinoma responds less well to chemotherapy and the difference in response rate between infiltrating lobular carcinoma and infiltrating duct carcinoma persisted even after adjusting for hormone-receptor status and the use of taxane.

Breasts harboring lobular neoplasia have been shown frequently to contain associated invasive lobular carcinoma and a high-risk duct lesion ${ }^{9}$ including atypical columnar cell changes ${ }^{36}$ and atypical cystic lobules. ${ }^{54}$ In general, some authors ${ }^{8,21}$ recommend that in patients with a diagnosis of lobular carcinoma in situ on core biopsy, a surgical excision is warranted if another high-risk lesion is present. Currently, the cases of in situ carcinoma with mixed lobular and ductal features are treated as duct carcinoma in situ. However, combined-type lesions with equivocal ductal and lobular features and absent or aberrant E-cadherin staining would be a challenge in clinical management.

In conclusion, this study indicates the followings: (1) for the most part, in breast lesions with equivocal histologic features of duct or lobular lesions, E-cadherin immunohistochemistry stain is a useful diagnostic tool to differentiate ductal from lobular carcinomas of the breast; (2) in general, tumors with a complete lack of E-cadherin membrane staining most likely represent lobular carcinoma, whereas tumors demonstrating circumferential membrane expression of E-cadherin invariably represent invasive carcinoma of the ductal type; (3) the most consistent changes of aberrant E-cadherin staining were partial thin linear staining or dot-like cytoplasmic staining in lobular lesions and reduced but thick membrane staining in duct lesions; (4) the most consistent morphologic changes for lobular neoplasia/infiltrating lobular carcinoma appear to be noncohesive uniform appearance and intracytoplasmic lumina of the tumor cells and (5) the classification of low-grade in situ lobular or duct 
carcinoma should not be based solely on the presence or absence of E-cadherin membrane activity due to presence of the existence of aberrant E-cadherin staining. The combination of E-cadherin staining and morphology is important for evaluation of a breast lesion with equivocal histologic features.

As the diagnosis of lobular neoplasia/infiltrating lobular carcinoma remains a highly subjective and challenging task, reviewing the cases with indeterminate morphology and/or E-cadherin staining in the intradepartmental conferences is of help in final interpretation of lobular neoplasia or duct neoplasia.

CK $8^{37}$ and high molecular weight cytokera$\operatorname{tin}^{17,55,56}$ have been used to distinguish between duct and lobular lesions, but we were not able to reproduce the same results. We are in the process of testing other immunomarkers such as catenins and other cadherin antibodies to distinguish lobular neoplasia or duct neoplasia.

There is a great need for the standardization in interpretation of E-cadherin immunostaining. In addition, microdissection of the lesions with indeterminate morphologic features and/or aberrant E-cadherin staining by utilizing emerging technologies such as high throughput genome mapping and microchip cDNA expression arrays ${ }^{46,48,49,52,57,58}$ may further elucidate molecular differences in these different types of breast cancer. Several of the genes identified in duct and lobular types may prove to be useful and may be attractive targets for therapies directed against specific types.

\section{References}

1 Foote FW, Stewart FW. Lobular carcinoma in situ. A rare form of mammary cancer. Am J Pathol 1941;17:491-499.

2 Haagensen CD, Lane $\mathrm{N}$, Lattes $\mathrm{R}$, et al. Lobular neoplasia (so-called lobular carcinoma in situ) of the breast. Cancer 1978;42:737-769.

3 Rogers LW. Carcinoma in situ (CIS). In: Page DL, Anderson TJ (eds). Diagnostic Histopathology of the Breast. Churchill Livingston: New York, NY, 1987, pp 157-197.

4 Rosen PP. Lobular carcinoma in situ and atypical lobular hyperplasia In: Rosen's Breast Pathology, 2nd edn. Lippincott William \& Wilkins: Philadelphia, PA, 2001, pp 581-626.

5 Schnitt SJ, Morrow M. Lobular carcinoma in situ: current concepts and controversies. Sem Diag Pathol 1999;16:209-223.

6 Tavassoli FA. Lobular neoplasia In: Tavassoli FA (ed). Pathology of the Breast, 2nd edn. McGraw-Hill: New York, NY, 1999, pp 373-400.

7 Rosen PP. Coexistent lobular carcinoma in situ and intraductal carcinoma in a single lobular-duct unit. Am J Surg Pathol 1980;4:241-246.

8 Fadare O, Dadmanesh F, Alvarado-Cabrero I, et al. Lobular intraepithelial neoplasia (lobular carcinoma in situ) with comedo-type necrosis: a clinicopathologic study of 18 cases. Am J Surg Pathol 2006;30:1445-1453.

9 Bratthauer GL, Tavassoli FA. Lobular intraepithelial neoplasia: previously unexplored aspects assessed in 775 cases and their clinical implications. Virchows Arch 2001;440:134-138.

10 Koerner F, Maluf H. Uncommon morphologic pattern of lobular neoplasia. Ann Diag Pathol 1999;4:249-259.

11 Arpino G, Bardou VJ, Clark GM, et al. Infiltrating lobular carcinoma of the breast: tumor characteristics and clinical outcome. Breast Cancer Res 2004;6:149-156.

12 Molland JG, Donnellan M, Janu NC, et al. Infiltrating lobular carcinoma-a comparison of diagnosis, management and outcome with infiltrating duct carcinoma. Breast 2004;13:389-396.

13 Peiro G, Bornstein BA, Connolly JL, et al. The influence of infiltrating lobular carcinoma on the outcome of patients treated with breast-conserving surgery and radiation therapy. Breast Cancer Res Treat 2000;59:49-54.

14 Siitonen SM, Kononen JT, Helin HJ, et al. Reduced E-cadherin expression is associated with invasiveness and unfavorable prognosis in breast cancer. Am J Clin Pathol 1996;105:394-402.

15 Acs G, Lawton TJ, Rebbeck TR, et al. Differential expression of E-cadherin in lobular and ductal neoplasms of the breast and its biologic and diagnostic implications. Am J Clin Pathol 2001;115:85-98.

16 Goldstein NS, Bassi D, Watts JC, et al. E-cadherin reactivity of 95 noninvasive ductal and lobular lesions of the breast. Implications for the interpretation of problematic lesions. Am J Clin Pathol 2001;115:534-542.

17 Wheeler DT, Tai LH, Bratthauer GL, et al. Tubulolobular carcinoma of the breast: an analysis of 27 cases of a tumor with a hybrid morphology and immunoprofile. Am J Surg Pathol 2004;28:1587-1593.

18 Rakha EA, Rehim AE, Pinder SE, et al. E-cadherin expression in invasive non-lobular carcinoma of the breast and its prognostic significance. Histopathology 2005;46:685-693.

19 Qureshi HS, Linden MD, Divine G, et al. E-cadherin status in breast cancer correlates with histologic type but does not correlate with established prognostic parameters. Am J Clin Pathol 2006;125:377-385.

20 Kuroda H, Tamaru J, Takeuchi I, et al. Expression of E-cadherin, $\alpha$-catenin, and $\beta$-catenin in tubulolobular carcinoma of the breast. Virchows Arch 2006;448: 500-505.

21 Jacobs TW. Recently recognized variants of lobular carcinoma in situ (LCIS) with an emphasis of management of LCIS on core needle biopsy. Pathol Case Rev 2003;8:211-219.

22 Moll R, Mitze M, Frixen UH, et al. Differential loss of E-cadherin expression in infiltrating ductal and lobular breast carcinoma. Am J Pathol 1993;143:1731-1742.

23 Asgeirsson KS, Jonasson JG, Tryggvadottir L, et al. Altered expression of E-cadherin in breast cancer: patterns, mechanisms and clinical significance. Eur J Cancer 2000;36:1098-1106.

24 Goldstein NS, Kestin LL, Vicini FA. Clinicopathologic implications of E-cadherin reactivity in patients with lobular carcinoma in situ of the breast. Cancer 2001;92:738-747.

25 Maluf HM, Swanson PE, Koerner FC. Solid low-grade in situ carcinoma of the breast: role of associated lesions and E-cadherin in differential diagnosis. Am J Surg Pathol 2001;25:237-244. 
26 Jacobs TW, Pliss N, Kouria G, et al. Carcinomas in situ of the breast with indeterminate features. Role of E-cadherin staining in categorization. Am J Surg Pathol 2001;25:229-236.

27 Goldstein NS. Does the level of E-cadherin expression correlate with the primary breast carcinoma infiltration pattern and type of systemic metastases? Am J Clin Pathol 2002;118:425-434.

28 Wahed A, Connelly J, Reese T. E-cadherin expression in pleomorphic lobular carcinoma: an aid to differentiation from ductal carcinoma. Ann Diag Pathol 2002;6:349-351.

29 Kowalski PJ, Rubin MA, Kleer CG. E-cadherin expression in primary carcinomas of the breast and its distant metastases. Breast Cancer Res 2003;5: R217-R222.

30 Kovacs A, Dhillon J, Walker RA. Expression of $\mathrm{P}$-cadherin, but not E-cadherin or $\mathrm{N}$-cadherin, relates to pathological and functional differentiation of breast carcinomas. BMJ 2003;56:318-322.

31 Harigopal M, Shin SJ, Murray MP, et al. Aberrant E-cadherin staining patterns in invasive mammary carcinoma. World J Surg Oncol 2005;3:73-85.

32 Bukholm IK, Nesland JM, Karesen R, et al. E-cadherin and $\alpha$-, $\beta$-, and $\gamma$-catenin protein expression in relation to metastasis in human breast carcinoma. J Pathol 1998;185:262-266.

33 Charpin C, Garcia S, Bonnier P, et al. Reduced E-cadherin immunohistochemical expression in node-negative breast carcinomas correlates with 10-year survival. Am J Clin Pathol 1997;109:431-438.

34 Oka H, Shizaki H, Kobayashi K, et al. Expression of E-cadherin cell adhesion molecules in human breast cancer tissues and its relationship to metastasis. Cancer Res 1993;53:1696-1701.

35 Yoshida R, Kimura N, Harada Y, et al. The loss of E-cadherin, alpha- and beta-catenin expression is associated with metastasis and poor prognosis in invasive carcinoma. Int J Oncol 2001;18:513-520.

36 Abdel-Fatah TM, Powe DG, Hodi Z, et al. High frequency of co-existence of columnar cell lesions, lobular neoplasia, and low grade ductal carcinoma in situ with invasive tubular carcinoma and invasive lobular carcinoma. Am J Surg Pathol 2007;31: 417-426.

37 Lehr HA, Folpe A, Yaziji $\mathrm{H}$, et al. Cytokeratin 8 immunostaining pattern and E-cadherin expression distinguish lobular from ductal breast carcinoma. Am J Clin Pathol 2000;114:190-196.

38 De Leeuw WJ, Berx G, Vos CB, et al. Simultaneous loss of E-cadherin and catenins in invasive lobular breast cancer and lobular carcinoma in situ. J Pathol 1997;183:404-411.

39 Hashizume R, Koizumi H, Ihara A, et al. Expression of $\beta$-catenin in normal breast tissue and breast carcinoma: a comparative study with epithelial cadherin and $\alpha$-catenin. Histopathology 1996;29:139-146.

40 Oyama T, Kanai TY, Ochiai A, et al. A truncated $\beta$-catenin disrupts the interaction between E-cadherin and $\alpha$-catenin: a cause of loss of intercellular adhesiveness in human cancer cell lines. Cancer Res 1994;54:6283-6287.

41 Ozawa M, Kemler R. Altered cell adhesion activity by pervanadate due to the dissociation of $\alpha$-catenin from the E-cadherin-catenin complex. J Biol Chem 1998; 273:6166-6170.
42 Steinberg MS, McNutt PM. Cadherins and their connections: adhesion junctions have broader functions. Curr Opin Cell Biol 1999;11:554-560.

43 Zschiesche W, Schpnborn I, Behrens J, et al. Expression of E-cadherin and catenins in invasive mammary carcinomas. Anticancer Res 1997;17:561-568.

44 Hwang ES, Nyante SJ, Chen YY, et al. Clonality of lobular carcinomas in situ and synchronous invasive lobular carcinoma. Cancer 2004;100:2562-2572.

45 Kanai Y, Oda T, Tsuda $\mathrm{H}$, et al. Point mutation of the E-cadherin gene in invasive carcinomas of the breast. Jpn J Cancer Res 1994;85:1035-1039.

46 Lerwill MF. The evolution of lobular neoplasia. Adv Anat Pathol 2006;13:157-165.

47 Sarrio D, Moreno-Bueno G, Hardisson D, et al. Epigenetic and genetic alterations of APC and CDHI genes in lobular breast cancer: relationships with abnormal E-cadherin and catenin expression and microsatellite instability. Int J Cancer 2003;106: 208-215.

48 Stange D, Radiwimmer B, Schubert F, et al. Highresolution genomic profiling reveals association ion of chromosomal aberration on $1 \mathrm{q}$ and $16 \mathrm{p}$ with histologic and genetic subgroups of invasive breast cancer. Clin Cancer Res 2006;12:345-352.

49 Xiaodong JI, Woodward AJ, Rimm DL, et al. Transcriptional defects underlie loss of E-cadherin expression in breast cancer. Cell Growth Differ 1997; 8:773-778

50 Caldeira JR, Prando EC, Quevedo FC, et al. CDH1 promotor hypermethylation and E-cadherin protein expression in infiltrating breast cancer. BMC Cancer 2006;6:48-60.

51 Graff JR, Herman JG, Lapidus RG, et al. E-cadherin expression is silenced by DNA hypermethylation in human breast and prostate carcinomas. Cancer Res 1995;55:5195-5199.

52 Vos CBJ, Cleton-Jansen AM, Berx G, et al. E-cadherin inactivation in lobular carcinoma in situ of the breast: an early event in tumorigenesis. $\mathrm{Br} \mathrm{J}$ Cancer 1997;76:1131-1133.

53 Cristofanilli M, Gonzalez-Angulo A, Sneige N, et al. Invasive lobular carcinoma classic type: response to primary chemotherapy and survival outcomes. J Clin Oncol 2005;23:41-48.

54 Brogi E, Oyama T, Koerner F. Atypical cystic lobules in patients with lobular neoplasia. Int J Surg Pathol 2001;9:201-206.

55 Berx G, Cleton-Jansen AM, Strumane K, et al. E-cadherin is inactivated in a majority of invasive human lobular breast cancers by truncation mutations throughout its extracellular domain. Oncogene 1996;13:1919-1925.

56 Bratthauer GL, Moinfar F, Stamatakos MD, et al. Combined E-cadherin and high molecular weight cytokeratin immunoprofile differentiates lobular, ductal and hybrid mammary epithelial neoplasias. Human Pathol 2002;33:620-627.

57 Korkola JE, De Vries S, Fridlyand J, et al. Differentiation of lobular versus ductal breast carcinomas by expression microarray analysis. Cancer Res 2003;63: 7167-7175.

58 Bempt IV, Vanhentenrijk V, Drijkoningen M, et al. Comparative expressed sequence hybridization reveals differential gene expression in morphological breast cancer subtypes. J Pathol 2006;208:486-494. 